\title{
DYNAMIC PROGRAMMING PRINCIPLE FOR TUG-OF-WAR GAMES WITH NOISE
}

\author{
Juan J. Manfredi ${ }^{1}$, Mikko Parviainen ${ }^{2}$ and Julio D. Rossi ${ }^{3}$
}

\begin{abstract}
We consider a two-player zero-sum-game in a bounded open domain $\Omega$ described as follows: at a point $x \in \Omega$, Players I and II play an $\varepsilon$-step tug-of-war game with probability $\alpha$, and with probability $\beta(\alpha+\beta=1)$, a random point in the ball of radius $\varepsilon$ centered at $x$ is chosen. Once the game position reaches the boundary, Player II pays Player I the amount given by a fixed payoff function $F$. We give a detailed proof of the fact that the value functions of this game satisfy the Dynamic Programming Principle
\end{abstract}

$$
u(x)=\frac{\alpha}{2}\left\{\sup _{y \in \bar{B}_{\varepsilon}(x)} u(y)+\inf _{y \in \bar{B}_{\varepsilon}(x)} u(y)\right\}+\beta f_{B_{\varepsilon}(x)} u(y) \mathrm{d} y,
$$

for $x \in \Omega$ with $u(y)=F(y)$ when $y \notin \Omega$. This principle implies the existence of quasioptimal Markovian strategies.

Mathematics Subject Classification. 35J70, 49N70, 91A15, 91A24.

Received December 1, 2009. Revised August 13, 2010.

Published online December 2, 2010.

\section{INTRODUCTION}

We study a two-player zero-sum-game described as follows: starting from a point $x_{0} \in \Omega$, Players I and II play the tug-of-war game with probability $\alpha$, and with probability $\beta$, a random point in $B_{\varepsilon}\left(x_{0}\right)$ is chosen. The players continue starting from the new point until the game position reaches a strip near the boundary, and then Player II pays Player I the amount given by a payoff function. This game was studied in a slightly different form by Peres and Sheffield in [8], and the tug-of-war game by Peres et al. in [9].

We aim to provide a self-contained proof of the Dynamic Programming Principle (DPP) written in a language more familiar to analysts. For a proof in the context of the general theory of stochastic games see the book of Maitra and Sudderth [4] in discrete state spaces, and the paper [3] for general state spaces.

Keywords and phrases. Dirichlet boundary conditions, Dynamic Programming Principle, $p$-Laplacian, stochastic games, twoplayer zero-sum games.

${ }^{1}$ Department of Mathematics, University of Pittsburgh, Pittsburgh, PA 15260, USA. manfredi@pitt.edu

2 Institute of Mathematics, Helsinki University of Technology, P.O. Box 1100, 02015 TKK, Finland. Mikko.Parviainen@tkk.fi

${ }^{3}$ Departamento de Matemática, FCEyN UBA (1428), Buenos Aires, Argentina. jrossi@dm.uba.ar 
To be more precise, we show that the value functions for the tug-of-war game with noise satisfy the following equation

$$
u(x)=\frac{\alpha}{2}\left\{\sup _{y \in \bar{B}_{\varepsilon}(x)} u(y)+\inf _{y \in \bar{B}_{\varepsilon}(x)} u(y)\right\}+\beta f_{B_{\varepsilon}(x)} u(y) \mathrm{d} y
$$

for $x \in \Omega$. This property provides a useful tool in many applications.

Intuitively, the expected payoff at the point can be calculated by summing up all the three cases: Player I moves, Player II moves, or a random point is chosen, with their corresponding probabilities. Player I, who tries to maximize the payoff, will choose a point maximizing the expected payoff, and Player II a point minimizing the expected payoff.

Functions satisfying the Dynamic Programming Principle with $\alpha=1$ and $\beta=0$, that is,

$$
u(x)=\frac{1}{2}\left\{\sup _{y \in \bar{B}_{\varepsilon}(x)} u(y)+\inf _{y \in \bar{B}_{\varepsilon}(x)} u(y)\right\}
$$

are called harmonious functions, see Le Gruyer [1] and Le Gruyer and Archer [2]. Furthermore, Oberman used a similar approach to obtain numerical approximations in [7]. As $\varepsilon$ goes to zero, harmonious functions approximate solutions to the infinity Laplacian. To be more precise, Le Gruyer proved in [1], see also [9], that a uniform limit of a sequence of harmonious functions when $\varepsilon \rightarrow 0$ is a solution to $\Delta_{\infty} u=0$, where

$$
\Delta_{\infty} u=|\nabla u|^{-2} \sum_{i j} u_{x_{i}} u_{x_{i} x_{j}} u_{x_{j}}=|\nabla u|^{-2}\left\langle D^{2} u \nabla u, \nabla u\right\rangle
$$

is the 1-homogeneous infinity Laplacian.

In the general case in [6], see also [5], the authors studied functions that satisfy (1.1) and proved that they approximate solutions to the $p$-Laplacian

$$
\operatorname{div}\left(|\nabla u|^{p-2} \nabla u\right)=0
$$

when

$$
\alpha=\frac{p-2}{p+n} \quad \text { and } \quad \beta=\frac{2+n}{p+n} \text {. }
$$

A key tool for the analysis was the Dynamic Programming Principle (1.1) applied to our game.

\section{TUG-OF-WAR GAMES WITH NOISE}

Let $\Omega \subset \mathbb{R}^{n}$ be a bounded open set and $\varepsilon>0$. We denote the compact boundary strip of width $\varepsilon$ by

$$
\Gamma_{\varepsilon}=\left\{x \in \mathbb{R}^{n} \backslash \Omega: \operatorname{dist}(x, \partial \Omega) \leq \varepsilon\right\}
$$

Further, we denote by $B_{\varepsilon}(x)$ the open Euclidean ball and with $\bar{B}_{\varepsilon}(x)$ its closure $\bar{B}_{\varepsilon}(x)=\left\{y \in \mathbb{R}^{n}:|y-x| \leq \varepsilon\right\}$.

Players I and II play as follows. At an initial time, they place a token at a point $x_{0} \in \Omega$ and toss a biased coin with probabilities $\alpha$ and $\beta, \alpha+\beta=1$. If they get heads (probability $\alpha$ ), they toss a fair coin and the winner of the toss moves the game position to any $x_{1} \in \bar{B}_{\varepsilon}\left(x_{0}\right)$ of his choice. On the other hand, if they get tails (probability $\beta$ ), the game state moves according to the uniform probability density to a random point $x_{1} \in B_{\varepsilon}\left(x_{0}\right)$. Then they continue playing from $x_{1}$. 
This procedure yields a sequence of game states $x_{0}, x_{1}, \ldots$ Once the game position reaches the boundary strip $\Gamma_{\varepsilon}$, the game ends and Player II pays Player I the amount given by a payoff function $F: \Gamma_{\varepsilon} \rightarrow \mathbb{R}$, which is assumed to be Borel measurable. Player I earns $F\left(x_{k}\right)$ while Player II earns $-F\left(x_{k}\right)$.

A strategy $S_{\mathrm{I}}$ for Player I is a collection of measurable mappings $S_{\mathrm{I}}=\left\{S_{\mathrm{I}}^{k}\right\}_{k=0}^{\infty}$ such that the next game position is

$$
S_{\mathrm{I}}^{k}\left(x_{0}, x_{1}, \ldots, x_{k}\right)=x_{k+1} \in \bar{B}_{\varepsilon}\left(x_{k}\right)
$$

if Player I wins the toss given a partial history $\left(x_{0}, x_{1}, \ldots, x_{k}\right)$. Similarly Player II plays according to a strategy $S_{\text {II }}$. The next game position $x_{k+1} \in \bar{B}_{\varepsilon}\left(x_{k}\right)$, given a partial history $\left(x_{0}, x_{1}, \ldots, x_{k}\right)$, is distributed according to the probability

$$
\pi_{S_{\mathrm{I}}, S_{\mathrm{II}}}\left(x_{0}, x_{1}, \ldots, x_{k}, A\right)=\frac{\beta\left|A \cap B_{\varepsilon}\left(x_{k}\right)\right|}{\left|B_{\varepsilon}\left(x_{k}\right)\right|}+\frac{\alpha}{2} \delta_{S_{\mathrm{I}}^{k}\left(x_{0}, x_{1}, \ldots, x_{k}\right)}(A)+\frac{\alpha}{2} \delta_{S_{\mathrm{II}}^{k}\left(x_{0}, x_{1}, \ldots, x_{k}\right)}(A) .
$$

From now on, we shall omit $k$ and simply denote the strategies by $S_{\mathrm{I}}$ and $S_{\mathrm{II}}$.

Let us fix strategies $S_{\mathrm{I}}, S_{\mathrm{II}}$. Let $\Omega_{\varepsilon}=\Omega \cup \Gamma_{\varepsilon} \subset \mathbb{R}^{n}$ be equipped with the natural topology, and the $\sigma$-algebra $\mathcal{B}$ of the Borel sets. The space of all game sequences

$$
H^{\infty}=\left\{x_{0}\right\} \times \Omega_{\varepsilon} \times \Omega_{\varepsilon} \times \ldots,
$$

is a product space endowed with the product topology.

Let $\left\{\mathcal{F}_{k}\right\}_{k=0}^{\infty}$ denote the filtration of $\sigma$-algebras, $\mathcal{F}_{0} \subset \mathcal{F}_{1} \subset \ldots$ defined as follows: $\mathcal{F}_{k}$ is the product $\sigma$-algebra generated by cylinder sets of the form $\left\{x_{0}\right\} \times A_{1} \times \ldots \times A_{k} \times \Omega_{\varepsilon} \times \Omega_{\varepsilon} \ldots$ with $A_{i} \in \mathcal{B}$. For

$$
\omega=\left(x_{0}, \omega_{1}, \ldots\right) \in H^{\infty},
$$

we define the coordinate processes

$$
X_{k}(\omega)=\omega_{k}, \quad X_{k}: H^{\infty} \rightarrow \mathbb{R}^{n}, k=0,1, \ldots
$$

so that $X_{k}$ is an $\mathcal{F}_{k}$-measurable random variable. Moreover, $\mathcal{F}_{\infty}=\sigma\left(\bigcup \mathcal{F}_{k}\right)$ is the smallest $\sigma$-algebra so that all $X_{k}$ are $\mathcal{F}_{\infty}$-measurable. To denote the time when the game state reaches the boundary, we define a random variable

$$
\tau(\omega)=\inf \left\{k: X_{k}(\omega) \in \Gamma_{\varepsilon}, k=0,1, \ldots\right\},
$$

which is a stopping time relative to the filtration $\left\{\mathcal{F}_{k}\right\}_{k=0}^{\infty}$.

A starting point $x_{0}$ and the strategies $S_{\text {I }}$ and $S_{\text {II }}$ determine a unique probability measure $\mathbb{P}_{S_{I}, S_{\text {II }}}^{x_{0}}$ on $H^{\infty}$ relative to the $\sigma$-algebra $\mathcal{F}^{\infty}$. This measure is built by Kolmogorov's extension theorem, see (3.2) below and Theorem 3.5 in Varadhan [10], to the family of transition probabilities

$$
\pi_{S_{\mathrm{I}}, S_{\mathrm{II}}}\left(x_{0}, X_{1}(\omega), \ldots, X_{k}(\omega), A\right)=\frac{\beta\left|A \cap B_{\varepsilon}\left(\omega_{k}\right)\right|}{\left|B_{\varepsilon}\left(\omega_{k}\right)\right|}+\frac{\alpha}{2} \delta_{S_{\mathrm{I}}\left(x_{0}, \omega_{1}, \ldots, \omega_{k}\right)}(A)+\frac{\alpha}{2} \delta_{S_{\mathrm{II}}\left(x_{0}, \omega_{1}, \ldots, \omega_{k}\right)}(A) .
$$

The expected payoff is then

$$
\mathbb{E}_{S_{\mathrm{I}}, S_{\mathrm{II}}}^{x_{0}}\left[F\left(X_{\tau}\right)\right]=\int_{H^{\infty}} F\left(X_{\tau}(\omega)\right) \mathbb{P}_{S_{\mathrm{I}}, S_{\mathrm{II}}}^{x_{0}}(\mathrm{~d} \omega)
$$

Note that, due to the fact that $\beta>0$, the game ends almost surely

$$
\mathbb{P}_{S_{\mathrm{I}}, S_{\mathrm{II}}}^{x_{0}}\left(\left\{\omega \in H^{\infty}: \tau(\omega)<\infty\right\}\right)=1
$$


for any choice of strategies because the game sequences contain arbitrary long sequences of random steps with probability 1.

The value of the game for Player $I$ is given by

$$
u_{\mathrm{I}}\left(x_{0}\right)=\sup _{S_{\mathrm{I}}} \inf _{S_{\mathrm{II}}} \mathbb{E}_{S_{\mathrm{I}}, S_{\mathrm{II}}}^{x_{0}}\left[F\left(X_{\tau}\right)\right]
$$

while the value of the game for Player II is given by

$$
u_{\mathrm{II}}\left(x_{0}\right)=\inf _{S_{\mathrm{II}}} \sup _{S_{\mathrm{I}}} \mathbb{E}_{S_{\mathrm{I}}, S_{\mathrm{II}}}^{x_{0}}\left[F\left(X_{\tau}\right)\right] .
$$

The values $u_{\mathrm{I}}\left(x_{0}\right)$ and $u_{\mathrm{II}}\left(x_{0}\right)$ are in a sense the best expected outcomes each player can almost guarantee when the game starts at $x_{0}$. For the measurability of the value functions we refer to [3].

\section{A PROOF OF THE DPP}

In this section, we prove that the values $u_{\mathrm{I}}$ and $u_{\mathrm{II}}$ satisfy the Dynamic Programming Principle. To this end, we define measures inductively on finite products as

$$
\begin{aligned}
& \mu_{S_{\mathrm{I}}, S_{\mathrm{II}}}^{0, x_{0}}\left(x_{0}\right)=1, \\
& \mu_{S_{\mathrm{I}}, S_{\mathrm{II}}}^{k, x_{\mathrm{I}}}\left(\left\{x_{0}\right\} \times A_{1} \times \ldots \times A_{k-1} \times A_{k}\right) \\
& =\int_{A_{1} \times \ldots \times A_{k-1}} \pi_{S_{\mathrm{I}}, S_{\mathrm{II}}}\left(x_{0}, \omega_{1}, \ldots, \omega_{k-1}, A_{k}\right) \mu_{S_{\mathrm{I}}, S_{\mathrm{II}}}^{k-1, x_{0}}\left(x_{0}, \mathrm{~d} \omega_{1}, \ldots, \mathrm{d} \omega_{k-1}\right) .
\end{aligned}
$$

This is the family of measures used to construct the measure $\mathbb{P}_{S_{I}, S_{\text {II }}}^{x_{0}}$ by applying Kolmogorov's theorem. In particular,

$$
\mu_{S_{\mathrm{I}}, S_{\mathrm{II}}}^{k, x_{0}}\left(\left\{x_{0}\right\} \times A_{1} \times \ldots \times A_{k}\right)=\mu_{S_{\mathrm{I}}, S_{\mathrm{II}}}^{k+1, x_{0}}\left(\left\{x_{0}\right\} \times A_{1} \times \ldots \times A_{k} \times \Omega_{\varepsilon}\right) .
$$

Denote $\mathcal{A}=\left\{x_{0}\right\} \times A_{1} \times \ldots \times A_{N} \times \Omega_{\varepsilon} \times \Omega_{\varepsilon} \times \ldots$ and $\mathcal{A}_{k}=\left\{x_{0}\right\} \times A_{1} \times \ldots \times A_{k}$. By Kolmogorov's extension theorem

$$
\mu_{S_{\mathrm{I}}, S_{\mathrm{II}}}^{k, x_{0}}\left(\mathcal{A}_{k}\right)=\mathbb{P}_{S_{I}, S_{\mathrm{II}}}^{x_{0}}(\mathcal{A}) \quad \text { for } k \geq N
$$

and thus

$$
\mathbb{P}_{S_{I}, S_{\text {II }}}^{x_{0}}(\mathcal{A})=\lim _{k \rightarrow \infty} \mu_{S_{\mathrm{I}}, S_{\text {II }}}^{k, x_{0}}\left(\mathcal{A}_{k}\right)
$$

Conditioning with respect to the possible first steps plays a key role in the proof. Following the notation in [3], we denote by $S_{\mathrm{I}}\left[x_{1}\right]$ a conditional strategy obtained by conditioning with respect to the first step. As the name suggests, this conditional strategy is defined on the partial histories of the form $\left(x_{0}, x_{1}, \ldots\right)$. Next we write the expectation as an integral of the conditional expectations.

Lemma 3.1. There exists a family of measures

$$
\left\{\mathbb{P}_{S_{I}\left[\omega_{1}\right], S_{I I}\left[\omega_{1}\right]}^{x_{0}, \omega_{1}}\right\}_{\omega_{1} \in \bar{B}_{\varepsilon}\left(x_{0}\right)}
$$

such that

$$
\begin{aligned}
\mathbb{E}_{S_{I}, S_{I I}}^{x_{0}}\left[F\left(X_{\tau}\right)\right] & =\int_{H^{\infty}} F\left(X_{\tau}(\omega)\right) \mathbb{P}_{S_{I}, S_{I I}}^{x_{0}}(\mathrm{~d} \omega) \\
& =\int_{\Omega_{\varepsilon}} \int_{\Omega_{\varepsilon} \times \ldots} F\left(X_{\tau}(\omega)\right) \mathbb{P}_{S_{I}\left[\omega_{1}\right], S_{I I}\left[\omega_{1}\right]}^{x_{0} \omega_{1}}\left(\mathrm{~d} \omega_{2}, \ldots\right) \pi_{S_{I}, S_{I I}}\left(x_{0}, \mathrm{~d} \omega_{1}\right) .
\end{aligned}
$$


The inner integral in the lemma above is a representative of a conditional expectation given the first points $x_{0}, X_{1}=X_{1}(\omega)=\omega_{1}$.

Proof of Lemma 3.1. First, observe that

$$
\mu_{S_{\mathrm{I}}, S_{\mathrm{II}}}^{1, x_{0}}\left(\left\{x_{0}\right\} \times A_{1}\right)=\pi_{S_{\mathrm{I}}, S_{\mathrm{II}}}\left(x_{0}, A_{1}\right) .
$$

Further,

$$
\begin{aligned}
\mu_{S_{\mathrm{I}}, S_{\mathrm{II}}}^{2, x_{\mathrm{I}}}\left(\left\{x_{0}\right\} \times A_{1} \times A_{2}\right) & =\int_{A_{1}} \pi_{S_{\mathrm{I}}, S_{\mathrm{II}}}\left(x_{0}, \omega_{1}, A_{2}\right) \pi_{S_{\mathrm{I}}, S_{\mathrm{II}}}\left(x_{0}, \mathrm{~d} \omega_{1}\right) \\
& =\int_{A_{1}} \int_{A_{2}} \tilde{\mu}_{S_{\mathrm{I}}\left[\omega_{1}\right], S_{\mathrm{II}}\left[\omega_{1}\right]}^{1,\left(x_{0}, \omega_{1}\right)}\left(\mathrm{d} \omega_{2}\right) \pi_{S_{\mathrm{I}}, S_{\mathrm{II}}}\left(x_{0}, \mathrm{~d} \omega_{1}\right),
\end{aligned}
$$

where the equality

$$
\tilde{\mu}_{S_{\mathrm{I}}\left[\omega_{1}\right], S_{\mathrm{II}}\left[\omega_{1}\right]}^{1,\left(x_{0}, A_{2}\right)}\left(A_{2}\right)=\pi_{S_{\mathrm{I}}, S_{\mathrm{II}}}\left(x_{0}, \omega_{1}, A_{2}\right)
$$

defines a new family of measures.

Now we assume, arguing by induction, that there exists $\tilde{\mu}_{S_{\mathrm{I}}\left[\omega_{1}\right], S_{\mathrm{II}}\left[\omega_{1}\right]}^{k-1,\left(x_{0}, \omega_{1}\right)}$ for $k=2,3, \ldots$ satisfying

$$
\mu_{S_{\mathrm{I}}, S_{\mathrm{II}}}^{k, x_{0}}\left(\left\{x_{0}\right\} \times \ldots \times A_{k}\right)=\int_{A_{1}} \int_{A_{2} \times \ldots \times A_{k}} \tilde{\mu}_{S_{\mathrm{I}}\left[\omega_{1}\right], S_{\mathrm{II}}\left[\omega_{1}\right]}^{k-1,\left(x_{0}, \omega_{1}\right)}\left(\mathrm{d} \omega_{2} \ldots, \mathrm{d} \omega_{k}\right) \pi_{S_{\mathrm{I}}, S_{\mathrm{II}}}\left(x_{0}, \mathrm{~d} \omega_{1}\right) .
$$

Next we show that this implies that there exists $\tilde{\mu}_{S_{\mathrm{I}}\left[\omega_{1}\right], S_{\mathrm{II}}\left[\omega_{1}\right]}^{k, x_{0}, \omega_{1}}$ satisfying a similar equality. Due to (3.4) this holds with $k=1$. To establish the inductive step, we utilize (3.1) together with (3.6), and calculate

$$
\begin{aligned}
\mu_{S_{\mathrm{I}}, S_{\mathrm{II}}}^{k+1, x_{0}}\left(\left\{x_{0}\right\} \times \ldots \times A_{k}\right) & =\int_{A_{1} \times \ldots \times A_{k}} \pi_{S_{\mathrm{I}}, S_{\mathrm{II}}}\left(x_{0}, \omega_{1}, \ldots, \omega_{k}, A_{k+1}\right) \mu_{S_{\mathrm{I}}, S_{\mathrm{II}}}^{k, x_{\mathrm{II}}}\left(x_{0}, \mathrm{~d} \omega_{1}, \ldots, \mathrm{d} \omega_{k}\right) \\
& =\int \pi_{S_{\mathrm{I}}, S_{\mathrm{II}}}\left(x_{0}, \omega_{1}, \ldots, \omega_{k}, A_{k+1}\right) \tilde{\mu}_{S_{\mathrm{I}}\left[\omega_{1}\right], S_{\mathrm{II}}\left[\omega_{1}\right]}^{k-1,\left(x_{0}, \omega_{1}\right)}\left(\mathrm{d} \omega_{2}, \ldots, \mathrm{d} \omega_{k}\right) \pi_{S_{\mathrm{I}}, S_{\mathrm{II}}}\left(x_{0}, \mathrm{~d} \omega_{1}\right) \\
& =\int_{A_{1}} \int_{A_{2} \times \ldots \times A_{k+1}} \tilde{\mu}_{S_{\mathrm{I}}\left[\omega_{1}\right], S_{\mathrm{II}}\left[\omega_{1}\right]}^{\left.k, x_{0}, \omega_{1}\right)}\left(\mathrm{d} \omega_{2} \ldots, \mathrm{d} \omega_{k+1}\right) \pi_{S_{\mathrm{I}}, S_{\mathrm{II}}}\left(x_{0}, \mathrm{~d} \omega_{1}\right)
\end{aligned}
$$

where the family of measures $\tilde{\mu}_{S_{\mathrm{I}}\left[\omega_{1}\right], S_{\mathrm{II}}\left[\omega_{1}\right]}^{k,\left(x_{0}, \omega_{1}\right)}$ is defined by

$$
\tilde{\mu}_{S_{\mathrm{I}}\left[\omega_{1}\right], S_{\mathrm{II}}\left[\omega_{1}\right]}^{k,\left(x_{2}, \omega_{1}\right)}\left(A_{2} \times \ldots \times A_{k+1}\right)=\int_{A_{2} \times \ldots \times A_{k}} \pi_{S_{\mathrm{I}}, S_{\mathrm{II}}}\left(x_{0}, \omega_{1}, \ldots, \omega_{k}, A_{k+1}\right) \tilde{\mu}_{S_{\mathrm{I}}\left[\omega_{1}\right], S_{\mathrm{II}}\left[\omega_{1}\right]}^{k-1,\left(x_{0}, \omega_{1}\right)}\left(\mathrm{d} \omega_{2}, \ldots, \mathrm{d} \omega_{k}\right) .
$$

Thus, we have inductively defined measures $\tilde{\mu}_{S_{\mathrm{I}}\left[\omega_{1}\right], S_{\mathrm{II}}\left[\omega_{1}\right]}^{\left.k, x_{0}, \omega_{1}\right)}$, which depend on the strategies $S_{\mathrm{I}}\left[\omega_{1}\right], S_{\mathrm{II}}\left[\omega_{1}\right]$, on the sets $A_{2} \times \ldots \times A_{k+1}$. By Kolmogorov's extension theorem, there exists a family of probability measures $\mathbb{P}_{S_{\mathrm{I}}\left[\omega_{1}\right], S_{\mathrm{II}}\left[\omega_{1}\right]}^{x_{0}, \omega_{1}}$ such that

$$
\mathbb{P}_{S_{\mathrm{I}}, S_{\mathrm{II}}}^{x_{0}}\left(\left\{x_{0}\right\} \times A_{1} \times \ldots\right)=\int_{A_{1}} \int_{A_{2} \times \ldots} \mathbb{P}_{S_{\mathrm{I}}\left[\omega_{1}\right], S_{\mathrm{II}}\left[\omega_{1}\right]}^{x_{0}, \omega_{1}}\left(\mathrm{~d} \omega_{2}, \ldots\right) \pi_{S_{\mathrm{I}}, S_{\mathrm{II}}}\left(x_{0}, \mathrm{~d} \omega_{1}\right) .
$$

The claim follows from this equation.

Every strategy $S_{\mathrm{I}}$ can be decomposed into the first step $S_{\mathrm{I}}\left(x_{0}\right)$ and the rest $S_{\mathrm{I}}^{\text {rest}}$, where $S_{\mathrm{I}}^{\text {rest }}$ contains exactly all the conditional strategies $S_{\mathrm{I}}\left[x_{1}\right], x_{1} \in \bar{B}_{\varepsilon}\left(x_{0}\right)$. The next lemma plays a key role in the proof of the DPP. 
Lemma 3.2. It holds that

$$
\sup _{S_{\mathrm{I}}\left(x_{0}\right)} \sup _{S_{\mathrm{I}}^{\text {rest }}} \inf _{S_{\mathrm{II}}\left(x_{0}\right)} \inf _{S_{\mathrm{II}}^{\text {rest }}} \mathbb{E}_{S_{\mathrm{I}}, S_{\mathrm{II}}}^{x_{\mathrm{II}}}\left[F\left(X_{\tau}\right)\right]=\sup _{S_{\mathrm{I}}\left(x_{0}\right)} \inf _{S_{\mathrm{II}}\left(x_{0}\right)} \sup _{S_{\mathrm{I}}^{\text {rest }}} \inf _{S_{\mathrm{II}}^{\text {rest }}} \mathbb{E}_{S_{\mathrm{I}}, S_{\mathrm{II}}}^{x_{0}}\left[F\left(X_{\tau}\right)\right] .
$$

Briefly, this is due to the fact that Player I can optimize her conditional strategy $S_{\mathrm{I}}[x]$ without affecting the conditional strategy $S_{\mathrm{I}}[y]$ if $y \neq x$. Thus we see that Player II gets no advantage by choosing his first step on the left hand side of (3.8).

Proof of Lemma 3.2. The direction " $\leq$ " is clear and we can concentrate on showing the reverse inequality. For any $\eta>0, \omega_{1} \in \bar{B}_{\varepsilon}\left(x_{0}\right)$, and $S_{\mathrm{I}}$, Player II can choose a strategy $S_{\mathrm{II}}^{*}\left[\omega_{1}\right]$ such that

$$
\int_{\Omega_{\varepsilon} \times \ldots} F\left(X_{\tau}(\omega)\right) \mathbb{P}_{S_{\mathrm{I}}\left[\omega_{1}\right], S_{\mathrm{II}}^{*}\left[\omega_{1}\right]}^{x_{0}, \omega_{1}}\left(\mathrm{~d} \omega_{2}, \ldots\right) \leq \inf _{S_{\mathrm{II}}\left[\omega_{1}\right]} \int_{\Omega_{\varepsilon} \times \ldots} F\left(X_{\tau}(\omega)\right) \mathbb{P}_{S_{\mathrm{I}}\left[\omega_{1}\right], S_{\mathrm{II}}\left[\omega_{1}\right]}^{x_{0}, \omega_{1}}\left(\mathrm{~d} \omega_{2}, \ldots\right)+\eta .
$$

Since the conditional strategy $S_{\mathrm{II}}[x]$ can be manipulated without affecting $S_{\mathrm{II}}[y]$ as long as $x \neq y$, this argument can be repeated for each $\omega_{1}$, and thus the procedure defines a strategy $S_{\mathrm{II}}^{*}, c f$. [3], so that the above inequality holds for each $\omega_{1} \in \bar{B}_{\varepsilon}\left(x_{0}\right)$ with this strategy. By using a trivial estimate and integrating the above inequality, we deduce

$$
\begin{aligned}
& \inf _{S_{\mathrm{II}}^{\text {rest }}} \int_{\Omega_{\varepsilon}} \int_{\Omega_{\varepsilon} \times \ldots} F\left(X_{\tau}(\omega)\right) \mathbb{P}_{S_{\mathrm{I}}\left[\omega_{1}\right], S_{\mathrm{II}}\left[\omega_{1}\right]}^{x_{0}, \omega_{1}}\left(\mathrm{~d} \omega_{2}, \ldots\right) \pi_{S_{\mathrm{I}}, S_{\mathrm{II}}}\left(x_{0}, \mathrm{~d} \omega_{1}\right) \\
& \leq \int_{\Omega_{\varepsilon}} \int_{\Omega_{\varepsilon} \times \ldots} F\left(X_{\tau}(\omega)\right) \mathbb{P}_{S_{\mathrm{I}}\left[\omega_{1}\right], S_{\mathrm{II}}^{*}\left[\omega_{1}\right]}^{x_{0}, \omega_{1}}\left(\mathrm{~d} \omega_{2}, \ldots\right) \pi_{S_{\mathrm{I}}, S_{\mathrm{II}}}\left(x_{0}, \mathrm{~d} \omega_{1}\right) \\
& \leq \int_{\Omega_{\varepsilon}}\left[\inf _{S_{\mathrm{II}}\left[\omega_{1}\right]} \int_{\Omega_{\varepsilon} \times \ldots} F\left(X_{\tau}(\omega)\right) \mathbb{P}_{S_{\mathrm{I}}\left[\omega_{1}\right], S_{\mathrm{II}}\left[\omega_{1}\right]}^{x_{0}, \omega_{1}}\left(\mathrm{~d} \omega_{2}, \ldots\right)\right] \pi_{S_{\mathrm{I}}, S_{\mathrm{II}}}\left(x_{0}, \mathrm{~d} \omega_{1}\right)+c \eta,
\end{aligned}
$$

for any $\pi_{S_{\mathrm{I}}, S_{\mathrm{II}}}$. We repeat the same argument for Player I with $\eta^{\prime}>0$, and construct $S_{\mathrm{I}}^{*}$. Thus recalling the previous inequality and (3.3), we deduce

$$
\begin{aligned}
\sup _{S_{\mathrm{I}}^{\text {rest }}} \inf _{S_{\mathrm{II}}^{\text {rest }}} \mathbb{E}_{S_{\mathrm{I}}, S_{\mathrm{II}}}^{x_{0}}\left[F\left(X_{\tau}\right)\right] & \leq \int_{\Omega_{\varepsilon}} \sup _{S_{\mathrm{I}}\left[\omega_{1}\right]} \inf _{S_{\mathrm{II}}\left[\omega_{1}\right]} \int_{\Omega_{\varepsilon} \times \ldots} F\left(X_{\tau}(\omega)\right) \mathbb{P}_{S_{\mathrm{I}}\left[\omega_{1}\right], S_{\mathrm{II}}\left[\omega_{1}\right]}^{x_{0}, \omega_{1}}\left(\mathrm{~d} \omega_{2}, \ldots\right) \pi_{S_{\mathrm{I}}, S_{\mathrm{II}}}\left(x_{0}, \mathrm{~d} \omega_{1}\right)+c \eta \\
& \leq \int_{\Omega_{\varepsilon}} \inf _{S_{\mathrm{II}}\left[\omega_{1}\right]} \int_{\Omega_{\varepsilon} \times \ldots} F\left(X_{\tau}(\omega)\right) \mathbb{P}_{S_{\mathrm{I}}^{*}\left[\omega_{1}\right], S_{\mathrm{II}}\left[\omega_{1}\right]}^{x_{0}, \omega_{1}}\left(\mathrm{~d} \omega_{2}, \ldots\right) \pi_{S_{\mathrm{I}}, S_{\mathrm{II}}}\left(x_{0}, \mathrm{~d} \omega_{1}\right)+c\left(\eta+\eta^{\prime}\right) \\
& \leq \inf _{S_{\mathrm{II}}^{\text {rest }}} \mathbb{E}_{S_{\mathrm{I}}^{*}, S_{\mathrm{II}}}^{x_{0}}\left[F\left(x_{\tau}\right)\right]+c\left(\eta+\eta^{\prime}\right) .
\end{aligned}
$$

Next we take $\inf _{S_{\text {II }}\left(x_{0}\right)}$ on both sides, use a trivial estimate, and obtain

$$
\begin{aligned}
\inf _{S_{\mathrm{II}}\left(x_{0}\right)} \sup _{S_{\mathrm{I}}^{\text {rest }}} \inf _{S_{\mathrm{II}}^{\text {rest }}} \mathbb{E}_{S_{\mathrm{I}}, S_{\mathrm{II}}}\left[F\left(X_{\tau}\right)\right] & \leq \inf _{S_{\mathrm{II}}\left(x_{0}\right)} \inf _{S_{\mathrm{II}}^{\text {rest }}} \mathbb{E}_{S_{\mathrm{I}}^{*}, S_{\mathrm{II}}}^{x_{0}}\left[F\left(X_{\tau}\right)\right]+c\left(\eta+\eta^{\prime}\right) \\
& \leq \sup _{S_{\mathrm{I}}^{\text {rest }}} \inf _{S_{\mathrm{II}}\left(x_{0}\right)} \inf _{S_{\mathrm{II}}^{\text {rest }}} \mathbb{E}_{S_{\mathrm{I}}, S_{\mathrm{II}}}^{x_{0}}\left[F\left(X_{\tau}\right)\right]+c\left(\eta+\eta^{\prime}\right) .
\end{aligned}
$$

Since $\eta, \eta^{\prime}>0$ are arbitrary, this proves (3.8).

Next we show that taking sup and inf over conditional strategies with respect to a fixed second point $\omega_{1}$ gives the value $u_{\mathrm{I}}$ at that point.

Lemma 3.3. Let $\omega_{1} \in \bar{B}_{\varepsilon}\left(x_{0}\right)$. We have

$$
u_{\mathrm{I}}\left(\omega_{1}\right)=\sup _{S_{\mathrm{I}}\left[\omega_{1}\right]} \inf _{S_{\mathrm{II}}\left[\omega_{1}\right]} \int_{\Omega_{\varepsilon} \times \ldots} F\left(X_{\tau}(\omega)\right) \mathbb{P}_{S_{\mathrm{I}}\left[\omega_{1}\right], S_{\mathrm{II}}\left[\omega_{1}\right]}^{x_{0}, \omega_{1}}\left(\mathrm{~d} \omega_{2}, \ldots\right) .
$$


Proof. We denote by $S_{\mathrm{I}}^{\omega_{1}}, S_{\mathrm{II}}^{\omega_{2}}$ strategies defined on $\left\{\omega_{1}\right\} \times \Omega_{\varepsilon} \times \Omega_{\varepsilon} \times \ldots$ i.e. we have a starting point $\omega_{1}$. Given such strategies $S_{\mathrm{I}}^{\omega_{1}}, S_{\mathrm{II}}^{\omega_{1}}$, we define conditional strategies $S_{\mathrm{I}}\left[\omega_{1}\right], S_{\mathrm{II}}\left[\omega_{1}\right]$ with the first points $x_{0}, \omega_{1}$ by setting

$$
S_{\mathrm{I}}\left[\omega_{1}\right]\left(x_{0}, \omega_{1}, \ldots, \omega_{k}\right)=S_{\mathrm{I}}^{\omega_{1}}\left(\omega_{1}, \ldots, \omega_{k}\right),
$$

and analogously for $S_{\mathrm{II}}^{\omega_{1}}$. Definition (3.10) applied in the calculation

$$
\begin{aligned}
\pi_{S_{\mathrm{I}}\left[\omega_{1}\right], S_{\mathrm{II}}\left[\omega_{1}\right]}\left(x_{0}, \omega_{1}, \ldots, \omega_{k}, A\right) & =\frac{\beta\left|A \cap B_{\varepsilon}\left(\omega_{k}\right)\right|}{\left|B_{\varepsilon}\left(\omega_{k}\right)\right|}+\frac{\alpha}{2} \delta_{S_{\mathrm{I}}\left[\omega_{1}\right]\left(x_{0}, \omega_{1}, \ldots, \omega_{k}\right)}(A)+\frac{\alpha}{2} \delta_{S_{\mathrm{II}}\left[\omega_{1}\right]\left(x_{0}, \omega_{1}, \ldots, \omega_{k}\right)}(A) \\
& =\frac{\beta\left|A \cap B_{\varepsilon}\left(\omega_{k}\right)\right|}{\left|B_{\varepsilon}\left(\omega_{k}\right)\right|}+\frac{\alpha}{2} \delta_{S_{\mathrm{I}}^{\omega_{1}}\left(\omega_{1}, \ldots, \omega_{k}\right)}(A)+\frac{\alpha}{2} \delta_{S_{\mathrm{II}}^{\omega_{1}}\left(\omega_{1}, \ldots, \omega_{k}\right)}(A) \\
& =\pi_{S_{\mathrm{I}}^{\omega_{1}}, S_{\mathrm{II}} \omega_{1}}\left(\omega_{1}, \ldots, \omega_{k}, A\right)
\end{aligned}
$$

shows that the transition probabilities are the same. This fact, (3.5), and (3.7) imply

$$
\begin{aligned}
\tilde{\mu}_{S_{\mathrm{I}}\left[\omega_{1}\right], S_{\mathrm{II}}\left[\omega_{1}\right]}^{2,\left(x_{0}, \omega_{1}\right)}\left(A_{2} \times A_{3}\right) & =\int_{A_{2}} \pi_{S_{\mathrm{I}}\left[\omega_{1}\right], S_{\mathrm{II}}\left[\omega_{1}\right]}\left(x_{0}, \omega_{1}, \omega_{2}, A_{3}\right) \tilde{\mu}_{S_{\mathrm{I}}\left[\omega_{1}\right], S_{\mathrm{II}}\left[\omega_{1}\right]}^{1,\left(x_{0}, \omega_{1}\right)}\left(\mathrm{d} \omega_{2}\right) \\
& =\int_{A_{2}} \pi_{S_{\mathrm{I}}\left[\omega_{1}\right], S_{\mathrm{II}}\left[\omega_{1}\right]}\left(x_{0}, \omega_{1}, \omega_{2}, A_{3}\right) \pi_{S_{\mathrm{I}}\left[\omega_{1}\right], S_{\mathrm{II}}\left[\omega_{1}\right]}\left(x_{0}, \omega_{1}, \mathrm{~d} \omega_{2}\right) \\
& =\int_{A_{2}} \pi_{S_{\mathrm{I}} \omega_{1}, S_{\mathrm{II}}^{\omega_{1}}}^{\omega_{1}}\left(\omega_{1}, \omega_{2}, A_{3}\right) \mu_{S_{\mathrm{I}} \omega_{1}, S_{\mathrm{II}}}^{1, \omega_{1}}\left(\omega_{1}, \mathrm{~d} \omega_{2}\right) \\
& =\mu_{S_{\mathrm{I}}^{2,}, \omega_{1}, S_{\mathrm{II}}}^{\omega_{1}}\left(\left\{\omega_{1}\right\} \times A_{2} \times A_{3}\right) .
\end{aligned}
$$

Assume then that

$$
\tilde{\mu}_{S_{\mathrm{I}}\left[\omega_{1}\right], S_{\mathrm{II}}\left[\omega_{1}\right]}^{k,\left(x_{0}, \omega_{1}\right)}\left(A_{2} \times \ldots \times A_{k+1}\right)=\mu_{S_{\mathrm{I}}^{\omega_{1}}, S_{\mathrm{II}}^{\omega_{1}}}^{k, \omega_{1}}\left(\left\{\omega_{1}\right\} \times A_{2} \times \ldots \times A_{k+1}\right) .
$$

This implies

$$
\begin{aligned}
\tilde{\mu}_{S_{\mathrm{I}}\left[\omega_{1}\right], S_{\mathrm{II}}\left[\omega_{1}\right]}^{k+1,\left(x_{0}, \omega_{1}\right)}\left(A_{2} \times \ldots \times A_{k+2}\right)= & \int_{A_{2} \times \ldots \times A_{k+1}} \pi_{S_{\mathrm{I}}\left[\omega_{1}\right], S_{\mathrm{II}}\left[\omega_{1}\right]}\left(x_{0}, \omega_{1}, \ldots, \omega_{k+1}, A_{k+2}\right) \\
& \times \tilde{\mu}_{S_{\mathrm{I}}\left[\omega_{1}\right], S_{\mathrm{II}}\left[\omega_{1}\right]}^{k,\left(\omega_{1}\right)}\left(\mathrm{d} \omega_{2}, \ldots, \mathrm{d} \omega_{k+1}\right) \\
= & \int_{A_{2} \times \ldots \times A_{k+1}} \pi_{S_{\mathrm{I}}^{\omega_{1}}, S_{\mathrm{II}}^{\omega_{1}}}\left(\omega_{1}, \ldots, \omega_{k+1}, A_{k+2}\right) \mu_{S_{\mathrm{I}}^{\omega_{1}}, S_{\mathrm{II}}^{\omega_{1}}}^{k, \omega_{1}}\left(\omega_{1}, \mathrm{~d} \omega_{2}, \ldots, \mathrm{d} \omega_{k+1}\right) \\
= & \mu_{S_{\mathrm{I}}^{\omega_{1}}, S_{\mathrm{II}}+1, \omega_{1}}^{\omega_{1}}\left(\left\{\omega_{1}\right\} \times A_{2} \times \ldots \times A_{k+2}\right) .
\end{aligned}
$$

Thus by induction

$$
\mathbb{P}_{S_{\mathrm{I}}\left[\omega_{1}\right], S_{\mathrm{II}}\left[\omega_{1}\right]}^{x_{0}, \omega_{1}}\left(A_{2} \times \ldots\right)=\mathbb{P}_{S_{\mathrm{I}}^{\omega_{1}}, S_{\mathrm{II}}^{\omega_{1}}}^{\omega_{1}}\left(\left\{\omega_{1}\right\} \times A_{2} \times \ldots\right) .
$$

Similarly, if we are given two conditional strategies $S_{\mathrm{I}}\left[\omega_{1}\right], S_{\mathrm{II}}\left[\omega_{1}\right]$, we can define strategies $S_{\mathrm{I}}^{\omega_{1}}, S_{\mathrm{II}}^{\omega_{1}}$, and repeat the above reasoning. By taking the infimum and the supremum, we obtain

$$
\sup _{S_{\mathrm{I}}\left[\omega_{1}\right]} \inf _{S_{\mathrm{II}}\left[\omega_{1}\right]} \int_{\Omega_{\varepsilon} \times \ldots} F\left(X_{\tau}(\omega)\right) \mathbb{P}_{S_{\mathrm{I}}\left[\omega_{1}\right], S_{\mathrm{II}}\left[\omega_{1}\right]}^{x_{0} \omega_{1}}\left(\mathrm{~d} \omega_{2}, \ldots\right)=\sup _{S_{\mathrm{I}}^{\omega_{1}}} \inf _{S_{\mathrm{II}}} \int_{\Omega_{\varepsilon} \times \Omega_{\varepsilon} \times \ldots} F\left(X_{\tau}\left(\omega_{1}, \ldots\right)\right) \mathbb{P}_{S_{\mathrm{I}}^{\omega_{1}}, S_{\mathrm{II}}^{\omega_{1}}}^{\omega_{1}}\left(\mathrm{~d} \omega_{2}, \ldots\right),
$$

which proves the claim.

Now, we are ready to prove our main result. 
Theorem 3.4 (DPP). The value function for Player I satisfies

$$
\begin{aligned}
& u_{\mathrm{I}}(x)=\frac{\alpha}{2}\left\{\sup _{y \in \bar{B}_{\varepsilon}(x)} u_{\mathrm{I}}(y)+\inf _{y \in \bar{B}_{\varepsilon}(x)} u_{\mathrm{I}}(y)\right\}+\beta f_{B_{\varepsilon}(x)} u_{\mathrm{I}}(y) \mathrm{d} y, \quad x \in \Omega, \\
& u_{\mathrm{I}}(x)=F(x), \quad x \in \Gamma_{\varepsilon} .
\end{aligned}
$$

Further, the value function for Player II satisfies the same equation.

Proof. The idea in the proof is to decompose the strategies with respect to the first step and the rest. Following the notation of Lemma 3.2, we have

$$
u_{\mathrm{I}}\left(x_{0}\right)=\sup _{S_{\mathrm{I}}} \inf _{S_{\mathrm{II}}} \mathbb{E}_{S_{\mathrm{I}}, S_{\mathrm{II}}}^{x_{0}}\left[F\left(X_{\tau}\right)\right]=\sup _{S_{\mathrm{I}}\left(x_{0}\right)} \sup _{S_{\mathrm{I}}^{\text {rest }}} \inf _{S_{\mathrm{II}}\left(x_{0}\right)} \inf _{S_{\mathrm{II}}^{\text {rest }}} \mathbb{E}_{S_{\mathrm{I}}, S_{\mathrm{II}}}^{x_{0}}\left[F\left(X_{\tau}\right)\right]
$$

According to Lemma 3.2

$$
\sup _{S_{\mathrm{I}}\left(x_{0}\right)} \sup _{S_{\mathrm{I}}^{\text {rest }}} \inf _{S_{\mathrm{II}}\left(x_{0}\right)} \inf _{S_{\mathrm{II}}^{\text {rest }}} \mathbb{E}_{S_{\mathrm{I}}, S_{\mathrm{II}}}^{x_{0}}\left[F\left(X_{\tau}\right)\right]=\sup _{S_{\mathrm{I}}\left(x_{0}\right)} \inf _{S_{\mathrm{II}}\left(x_{0}\right)} \sup _{S_{\mathrm{I}}^{\text {rest }}} \inf _{S_{\mathrm{II}}^{\text {rest }}} \mathbb{E}_{S_{\mathrm{I}}, S_{\mathrm{II}}}^{x_{0}}\left[F\left(X_{\tau}\right)\right] .
$$

This together with (3.3) implies

$$
\begin{aligned}
u_{\mathrm{I}}\left(x_{0}\right) & =\sup _{S_{\mathrm{I}}\left(x_{0}\right)} \inf _{S_{\mathrm{II}}\left(x_{0}\right)} \sup _{S_{\mathrm{I}}^{\text {rest }}} \inf _{S_{\mathrm{II}}^{\text {rest }}} \mathbb{E}_{S_{\mathrm{I}}, S_{\mathrm{II}}}^{x_{0}}\left[F\left(X_{\tau}\right)\right] \\
& =\sup _{S_{\mathrm{I}}\left(x_{0}\right)} \inf _{S_{\mathrm{II}}\left(x_{0}\right)} \int_{\Omega_{\varepsilon}} V\left(F\left(x_{\tau}\right) \mid x_{0}, \omega_{1}\right) \pi_{S_{\mathrm{I}}, S_{\mathrm{II}}}\left(x_{0}, \mathrm{~d} \omega_{1}\right),
\end{aligned}
$$

where we denoted

$$
V\left(F\left(x_{\tau}\right) \mid x_{0}, \omega_{1}\right)=\sup _{S_{\mathrm{I}}\left[\omega_{1}\right]} \inf _{S_{\mathrm{II}}\left[\omega_{1}\right]} \int_{\Omega_{\varepsilon} \times \ldots} F\left(X_{\tau}(\omega)\right) \mathbb{P}_{S_{\mathrm{I}}\left[\omega_{1}\right], S_{\mathrm{II}}\left[\omega_{1}\right]}^{x_{1} \omega_{1}}\left(\mathrm{~d} \omega_{2}, \ldots\right) .
$$

The justification for interchanging sup inf with the first integral is similar to that in (3.8), that is, the conditional strategy $S_{\mathrm{I}}[x]$ can be manipulated without affecting $S_{\mathrm{I}}[y]$ as long as $x \neq y$. By (3.11), Lemma 3.3, and (2.1), we obtain

$$
\begin{aligned}
u_{\mathrm{I}}\left(x_{0}\right) & =\sup _{S_{\mathrm{I}}\left(x_{0}\right)} \inf _{S_{\mathrm{II}}\left(x_{0}\right)} \int_{\Omega_{\varepsilon}} u_{\mathrm{I}}\left(\omega_{1}\right) \pi_{S_{\mathrm{I}}, S_{\mathrm{II}}}\left(x_{0}, \mathrm{~d} \omega_{1}\right) \\
& =\sup _{S_{\mathrm{I}}\left(x_{0}\right)} \inf _{S_{\mathrm{II}}\left(x_{0}\right)}\left[\frac{\alpha}{2}\left\{u_{\mathrm{I}}\left(S_{\mathrm{I}}\left(x_{0}\right)\right)+u_{\mathrm{I}}\left(S_{\mathrm{II}}\left(x_{0}\right)\right)\right\}+\beta f_{B_{\varepsilon}\left(x_{0}\right)} u_{\mathrm{I}} \mathrm{d} y\right] \\
& =\frac{\alpha}{2}\left\{\sup _{S_{\mathrm{I}}\left(x_{0}\right)} u_{\mathrm{I}}\left(S_{\mathrm{I}}\left(x_{0}\right)\right)+\inf _{S_{\mathrm{II}}\left(x_{0}\right)} u_{\mathrm{I}}\left(S_{\mathrm{II}}\left(x_{0}\right)\right)\right\}+\beta f_{B_{\varepsilon}\left(x_{0}\right)} u_{\mathrm{I}} \mathrm{d} y .
\end{aligned}
$$

In the last equality we used the fact that the infimum only affects the second term, the supremum only the first term, and the third term is independent of the strategies. This completes the proof.

Let the payoff function $F$ be bounded. Given the value functions

$$
u_{\mathrm{I}}\left(x_{0}\right)=\sup _{S_{\mathrm{I}}} \inf _{S_{\mathrm{II}}} \mathbb{E}_{S_{\mathrm{I}}, S_{\mathrm{II}}}^{x_{0}}\left[F\left(X_{\tau}\right)\right]
$$


and

$$
u_{\mathrm{II}}\left(x_{0}\right)=\inf _{S_{\mathrm{II}}} \sup _{S_{\mathrm{I}}} \mathbb{E}_{S_{\mathrm{I}}, S_{\mathrm{II}}}^{x_{0}}\left[F\left(X_{\tau}\right)\right],
$$

there are natural strategies for each player. These strategies turn out to be Markovian and quasioptimal. Fix $\eta>0$ and define strategies

$$
\check{S}_{\mathrm{I}}=\left\{\check{S}_{\mathrm{I}}^{k}\right\}_{k=0}^{\infty} \quad \text { and } \quad \check{S}_{\mathrm{II}}=\left\{\check{S}_{\mathrm{II}}^{k}\right\}_{k=0}^{\infty}
$$

as follows:

$$
\begin{aligned}
& \check{S}_{\mathrm{I}}^{k}\left(x_{0}, x_{1}, \ldots, x_{k}\right)=x_{k+1} \in \bar{B}_{\varepsilon}\left(x_{k}\right), \text { where } u\left(x_{k+1}\right) \geq \sup _{y \in \bar{B}_{\varepsilon}\left(x_{k}\right)} u(y)-2^{-k} \eta \\
& \check{S}_{\mathrm{II}}^{k}\left(x_{0}, x_{1}, \ldots, x_{k}\right)=x_{k+1} \in \bar{B}_{\varepsilon}\left(x_{k}\right), \text { where } u\left(x_{k+1}\right) \leq \inf _{y \in \bar{B}_{\varepsilon}\left(x_{k}\right)} u(y)+2^{-k} \eta .
\end{aligned}
$$

These strategies depend on $\eta$ and they define a measure $\mathbb{P}_{\tilde{S}_{\mathrm{I}}, \tilde{S}_{\text {II }}}^{x_{0}, \eta}$ on $\mathcal{F}^{\infty}$. With respect to this measure the value corresponding to a game with fixed strategies $\breve{S}_{\mathrm{I}}$ and $\breve{S}_{\mathrm{II}}$ is given by

$$
v_{\eta}\left(x_{0}\right)=\mathbb{E}_{\check{S}_{\mathrm{I}}, \breve{S}_{\mathrm{II}}}^{x_{0}, \eta}\left[F\left(X_{\tau}\right)\right] .
$$

Theorem 3.5. It holds that

$$
\left|u_{I}\left(x_{0}\right)-v_{\eta}\left(x_{0}\right)\right| \leq \eta
$$

Proof. The proof is based on the following observation

$$
\begin{aligned}
\mathbb{E}_{S_{\mathrm{I}}, \check{S ̆}_{\mathrm{II}}}^{x_{0}}\left[u_{\mathrm{I}}\left(x_{k}\right)+\eta 2^{-k} \mid x_{0}, \ldots, x_{k-1}\right] & \leq \frac{\alpha}{2}\left\{\inf _{\bar{B}_{\varepsilon}\left(x_{k-1}\right)} u_{\mathrm{I}}+\eta 2^{-k}+\sup _{\bar{B}_{\varepsilon}\left(x_{k-1}\right)} u_{\mathrm{I}}\right\}+\beta f_{B_{\varepsilon}\left(x_{k-1}\right)} u_{\mathrm{I}} \mathrm{d} y+\eta 2^{-k} \\
& \leq u_{\mathrm{I}}\left(x_{k-1}\right)+\eta 2^{-(k-1)},
\end{aligned}
$$

where we have estimated the strategy of Player I by the supremum and used the definition of $\check{S}_{\text {II }}$. Therefore we conclude that

$$
u_{\mathrm{I}}\left(x_{k}\right)+\eta 2^{-k}
$$

is a supermartingale. Since $u_{\mathrm{I}}=v_{\eta}=F$ at the boundary strip $\Gamma_{\varepsilon}$, we deduce

$$
\begin{aligned}
v_{\eta}\left(x_{0}\right) & =\mathbb{E}_{\check{S}_{\mathrm{I}}, \check{S}_{\mathrm{II}}}^{x_{0}}\left[F\left(x_{\tau}\right)\right]=\mathbb{E}_{\check{S}_{\mathrm{I}}, \breve{S}_{\mathrm{II}}}^{x_{0}}\left[v_{\eta}\left(x_{\tau}\right)\right] \\
& =\mathbb{E}_{\check{S}_{\mathrm{I}}, \breve{S}_{\mathrm{II}}}^{x_{\mathrm{I}}}\left[u_{\mathrm{I}}\left(x_{\tau}\right)\right]=\mathbb{E}_{\breve{S}_{\mathrm{I}}, \breve{S}_{\mathrm{II}}}^{x_{\mathrm{II}}}\left[u_{\mathrm{I}}\left(x_{\tau}\right)+\eta 2^{-\tau}\right]-\mathbb{E}_{\breve{S}_{\mathrm{I}}, \breve{S}_{\mathrm{II}}}^{x_{0}}\left[\eta 2^{-\tau}\right] \\
& \leq \mathbb{E}_{\breve{S}_{\mathrm{I}}, \breve{S}_{\mathrm{II}}}\left[u_{\mathrm{I}}\left(x_{\tau}\right)+\eta 2^{-\tau}\right] \\
& \leq \liminf _{k \rightarrow \infty} \mathbb{E}_{\breve{S}_{\mathrm{I}}, \breve{S}_{\mathrm{II}}}\left[u_{\mathrm{I}}\left(x_{\tau \wedge k}\right)+\eta 2^{-\tau \wedge k}\right] \\
& \leq u_{\mathrm{I}}\left(x_{0}\right)+\eta,
\end{aligned}
$$

where $\tau \wedge k=\min (\tau, k)$, and we used Fatou's lemma as well as the optional stopping theorem for $u_{\mathrm{I}}\left(x_{k}\right)+\eta 2^{-k}$.

This shows that $u_{\mathrm{I}}\left(x_{0}\right)-v_{\eta}\left(x_{0}\right) \geq-\eta$. The inequality $u_{\mathrm{I}}\left(x_{0}\right)-v_{\eta}\left(x_{0}\right) \leq \eta$ follows by a symmetric argument using $\check{S}_{\mathrm{I}}$.

Acknowledgements. Part of this work was done during a visit of M.P. and J.D.R. to University of Pittsburgh. They wish to thank for the friendly and stimulating atmosphere found there. J.D.R. partially supported by project MTM2004-02223, MEC, Spain, by UBA X066 and by CONICET, Argentina. J.J.M. supported in part by NSF award DMS-1001179. 


\section{REFERENCES}

[1] E. Le Gruyer, On absolutely minimizing Lipschitz extensions and PDE $\Delta_{\infty}(u)=0$. NoDEA 14 (2007) $29-55$

[2] E. Le Gruyer and J.C. Archer, Harmonious extensions. SIAM J. Math. Anal. 29 (1998) 279-292.

[3] A.P. Maitra and W.D. Sudderth, Borel stochastic games with lim sup payoff. Ann. Probab. 21 (1993) 861-885.

[4] A.P. Maitra and W.D. Sudderth, Discrete gambling and stochastic games, Applications of Mathematics 32. Springer-Verlag (1996).

[5] J.J. Manfredi, M. Parviainen and J.D. Rossi, An asymptotic mean value property characterization of $p$-harmonic functions. Proc. Am. Math. Soc. 138 (2010) 881-889.

[6] J.J. Manfredi, M. Parviainen and J.D. Rossi, On the definition and properties of p-harmonious functions. Preprint (2009).

[7] A. Oberman, A convergent difference scheme for the infinity Laplacian: construction of absolutely minimizing Lipschitz extensions. Math. Comp. 74 (2005) 1217-1230.

[8] Y. Peres and S. Sheffield, Tug-of-war with noise: a game theoretic view of the p-Laplacian. Duke Math. J. 145 (2008) 91-120.

[9] Y. Peres, O. Schramm, S. Sheffield and D. Wilson, Tug-of-war and the infinity Laplacian. J. Am. Math. Soc. 22 (2009) 167-210.

[10] S.R.S. Varadhan, Probability theory, Courant Lecture Notes in Mathematics 7. Courant Institute of Mathematical Sciences, New York University/AMS (2001). 